Commun. Math. Phys. 147, 199-210 (1992)

Communications in

Mathematical

Physics

(C) Springer-Verlag 1992

\title{
Realizability of a Model in Infinite Statistics
}

\section{Don Zagier}

Max-Planck-Institut für Mathematik, Bonn, FRG and University of Maryland, College Park, MD 20742, USA

Received August 26, 1991; in revised form November 22, 1991

\begin{abstract}
Following Greenberg and others, we study a space with a collection of operators $a(k)$ satisfying the " $q$-mutator relations" $a(l) a^{\dagger}(k)-q a^{\dagger}(k) a(l)=\delta_{k, l}$ (corresponding for $q= \pm 1$ to classical Bose and Fermi statistics). We show that the $n ! \times n$ ! matrix $A_{n}(q)$ representing the scalar products of $n$-particle states is positive definite for all $n$ if $q$ lies between -1 and +1 , so that the commutator relations have a Hilbert space representation in this case (this has also been proved by Fivel and by Bozejko and Speicher). We also give an explicit factorization of $A_{n}(q)$ as a product of matrices of the form $\left(1-q^{j} T\right)^{ \pm 1}$ with $1 \leqq j \leqq n$ and $T$ a permutation matrix. In particular, $A_{n}(q)$ is singular if and only if $q^{M}=1$ for some integer $M$ of the form $k^{2}-k, 2 \leqq k \leqq n$.
\end{abstract}

\section{Introduction}

In this paper we study the following object: a Hilbert space $\mathbf{H}$ together with a nonzero distinguished vector $|0\rangle$ (vacuum state) and a collection of operators $a_{k}$ : $\mathbf{H} \rightarrow \mathbf{H}$ satisfying the commutation relations (" $q$-mutator relations")

$$
a(l) a^{\dagger}(k)-q a^{\dagger}(k) a(l)=\delta_{k, l} \quad(\forall k, l)
$$

and the relations

$$
a(k)|0\rangle=0 \quad(\forall k) .
$$

Here $q$ is a fixed real number and $a^{\dagger}(l)$ denotes the adjoint of $a(l)$. The statistics based on the commutation relation (1) generalizes classical Bose and Fermi statistics, corresponding to $q=1$ and $q=-1$, respectively, as well as the intermediate case $q=0$ suggested by Hegstrom and investigated by Greenberg [1]. The study of the general case was inititated by Polyakov and Biedenharn [2].

Our first main result is a realizability theorem saying that the object just described exists if $-1<q<1$. In view of (2), we can think of the $a(k)$ as annihilation operators and the $a^{\dagger}(k)$ as creation operators. As well as the 0particle state $|0\rangle$, our space must contain the many-particle states obtained by applying combinations of $a(k)$ 's and $a^{\dagger}(k)$ 's to $|0\rangle$. To prove the realizability of our model it is obviously necessary and sufficient to consider the minimal space 
containing these vectors. We therefore define for each $q \in \mathbb{R}$ an inner product space $\mathbf{H}(q)$ generated by $|0\rangle$ and its images under polynomials in the operators $a(k)$ and $a^{\dagger}(k)$, subject to the relations (1) and (2). It has a basis consisting of $n$-particle states

$$
\mathbf{x}_{\mathrm{k}}=a^{\dagger}\left(k_{1}\right) \cdots a^{\dagger}\left(k_{n}\right)|0\rangle
$$

for each $n \geqq 0$ and each $n$-tuple of indices $\mathbf{k}=\left(k_{1}, \ldots, k_{n}\right)$, since we can use (1) to write any monomial in the $a(k)$ 's and $a^{\dagger}(k)$ 's as a sum of monomials having all the $a(k)$ 's on the right and all the $a^{\dagger}(k)$ 's on the left, and the only ones of these which do not annihilate $|0\rangle$ are those consisting of $a^{\dagger}(k)$ 's only (the linear independence is clear). By the same argument, we can use (1) and (2) to calculate each scalar product $\left(\mathbf{x}_{\mathbf{l}}, \mathbf{x}_{\mathbf{k}}\right)$ as a polynomial in $q$, for instance, for $k \neq l$ we have

$$
\begin{aligned}
\left(\mathbf{x}_{k l}, \mathbf{x}_{l k}\right) & =\left\langle 0\left|a(l) a(k) a^{\dagger}(l) a^{\dagger}(k)\right| 0\right\rangle=q\left\langle 0\left|a(l) a^{\dagger}(l) a(k) a^{\dagger}(k)\right| 0\right\rangle \\
& =q\left\langle 0\left|\left(1+q a^{\dagger}(l) a(l)\right)\left(1+q a^{\dagger}(k) a(k)\right)\right| 0\right\rangle=q\langle 0 \mid 0\rangle=q .
\end{aligned}
$$

(Here $\langle 0|$ denotes the operator $(|0\rangle, \cdot)$ and we have normalized by $\langle 0 \mid 0\rangle=1$.) In particular, for each value of $q$ the infinite matrix $A(q)=\left\{\left(x_{1}, x_{\mathbf{k}}\right)\right\}_{1, \mathbf{k}}$ is welldefined. The condition for the Hilbert space realizability of the $q$-mutator relation (1) is then that $A(q)$ be positive definite, i.e., that $(\mathbf{x}, \mathbf{x})>0$ for every non-zero vector $\mathbf{x} \in \mathbf{H}(q)$.

Theorem 1. The matrix $A(q)$ is positive definite for $-1<q<1$, so that the $q$ mutator relation (1) has a Hilbert space realization for $q$ in this range.

It is easy to see that $\left(x_{\mathbf{k}}, x_{1}\right)$ vanishes unless $\mathbf{k}$ is a permutation of $\mathbf{l}$. Thus the space $\mathbf{H}(q)$ [respectively the matrix $A(q)$ ] is the direct sum of infinitely many finitedimensional spaces (respectively matrices) indexed by all unordered $n$-tuples $\left\{k_{1}, \ldots, k_{n}\right\}$, and we only have to show the positive definiteness of these. We will show in Sect. 2 that the general case of this follows from the case when all of the indices $k_{i}$ are distinct. It is not hard to see (Sect. 2) that

$$
\left(\mathbf{x}_{\pi(1) \ldots \pi(n)}, \mathbf{x}_{1 \ldots n}\right)=q^{I(\pi)}
$$

for each permutation $\pi$ in the $n^{\text {th }}$ symmetric group $\Xi_{n}$, where $I(\pi)$ denotes the number of inversions of $\pi$, i.e., the number of $i, j \in[1, n]$ for which $i<j$ but $\pi(i)>\pi(j)$. Thus the problem reduces to showing that the $n ! \times n !$ matrix $A_{n}=A_{n}(q)$ defined by

$$
A_{n}(\pi, \sigma)=q^{\left.I / \sigma^{-1} \pi\right)} \quad\left(\pi, \sigma \in \Xi_{n}\right)
$$

is positive definite for $q$ between -1 and 1 . For this, in turn, it is sufficient by continuity to show that $A_{n}(q)$ is non-singular in this range, since $A_{n}(0)$ is the identity matrix and the eigenvalues of $A_{n}(q)$ vary continuously with $q$ and are real for $q$ real (because $A_{n}(q)$ is real and symmetric). We will prove the following stronger statement.

Theorem 2. The determinant of the matrix $A_{n}(q)$ is given by

$$
\operatorname{det} A_{n}(q)=\prod_{k=1}^{n-1}\left(1-q^{k^{2}+k}\right)^{\frac{n !(n-k)}{k^{2}+k}} .
$$

In particular, $A_{n}(q)$ is non-singular for all complex numbers $q$ except the $N^{\text {th }}$ roots of unity for $N=2,6,12, \ldots, n^{2}-n$. 
We will also describe explicitly the inverse of $A_{n}(q)$. Based on calculations for $n \leqq 5$, we conjecture that

$$
A_{n}(q)^{-1} \stackrel{?}{\in} \frac{1}{\Delta_{n}} M_{n !}(\mathbb{Z}[q]), \quad \Delta_{n}:=\prod_{k=1}^{n-1}\left(1-q^{k^{2}+k}\right) .
$$

For instance, for $n=3$ we have

$$
A_{3}(q)=\left(\begin{array}{cccccc}
1 & q & q & q^{2} & q^{2} & q^{3} \\
q & 1 & q^{2} & q & q^{3} & q^{2} \\
q & q^{2} & 1 & q^{3} & q & q^{2} \\
q^{2} & q & q^{3} & 1 & q^{2} & q \\
q^{2} & q^{3} & q & q^{2} & 1 & q \\
q^{3} & q^{2} & q^{2} & q & q & 1
\end{array}\right)
$$

where the rows and columns are indexed by the elements of $\Theta_{3}$ in the order [123], [213], [132], [231], [312], [321] (we use $\left[j_{1} \ldots j_{n}\right]$ to denote the element $\pi$ of $\mathfrak{S}_{n}$ defined by $\left.\pi(i)=j_{i}\right)$. The determinant of this matrix is $\left(1-q^{2}\right)^{6}\left(1-q^{6}\right)$ and its inverse is

$$
A_{3}(q)^{-1}=\Delta_{3}^{-1}\left(\begin{array}{cccccc}
1+q^{2} & -q & -q & -q^{4} & -q^{4} & q^{3}+q^{5} \\
-q & 1+q^{2} & -q^{4} & -q & q^{3}+q^{5} & -q^{4} \\
-q & -q^{4} & 1+q^{2} & q^{3}+q^{5} & -q & -q^{4} \\
-q^{4} & -q & q^{3}+q^{5} & 1+q^{2} & -q^{4} & -q \\
-q^{4} & q^{3}+q^{5} & -q & -q^{4} & 1+q^{2} & -q \\
q^{3}+q^{5} & -q^{4} & -q^{4} & -q & -q & 1+q^{2}
\end{array}\right)
$$

Finally, we remark that the matrix $A_{n}(q)$ splits as a direct sum of pieces corresponding to the irreducible representations of $\Xi_{n}$, the piece corresponding to a representation $\Pi I$ of dimension $d$ being the direct sum of $d$ copies of a $d \times d$ matrix $A_{n, I}(q)$. For the bosonic and fermionic cases $q=1$ and $q=-1$ all of these matrices are identically zero except for the one corresponding to the onedimensional trivial or alternating representation, respectively, but for $-1<q<1$ Theorem 1 says that every representation of every symmetric group occurs in a non-trivial (indeed, non-degenerate) way. (This is the reason for the term "infinite statistics" used by the physicists.) It would be of interest to calculate the determinants of the matrices $A_{n, \Pi}(q)$, say in terms of the Young diagram corresponding to $\Pi$. By Theorem 2 , each of these determinants is a product of cyclotomic polynomials $\Phi_{m}(q)$ for integers $m$ dividing some $k^{2}+k$, $1 \leqq k \leqq n-1$.

The paper is organized as follows. In Sect. 2 we give some generalities on group determinants and show that Theorem 1 follows from Theorem 2, which is then proved in Sect. 3. In Sect. 4 we give an explicit description of the inverse matrix of $A_{n}(q)$, while Sect. 5 gives a conjectural formula for the "number operators" in the Hilbert space $\mathbf{H}(q)$.

The author would like to thank O.W. Greenberg who told him about the $q$ mutator relation and suggested the problem of proving the positive definiteness for $-1<q<1$. This positive definiteness has been proved independently by Fivel and by Bozejko and Speicher [3]. (However, Fivel apparently asserts that the zeros of $A_{n}(q)$ are all roots of $q^{2 n}=1$, which contradicts Theorem 2 and is false for all 
$n \geqq 4$.) Consequences and related results are discussed in several subsequent papers by Greenberg [4].

\section{Group Determinants and the Reduction to $A_{n}(q)$}

Let $G$ be a finite group of order $m$ and $\varrho: G \rightarrow G L(V)$ a representation of $G$ on a (finite-dimensional) complex vector space $V$. We can extend $\varrho$ to an algebra homomorphism from the group algebra

$$
\mathbb{C}[G]=\left\{\sum_{g \in G} t_{g} g \mid t_{g} \in \mathbb{C} \text { for } g \in G\right\}
$$

to the matrix algebra End $(V)$ by $\varrho\left(\sum t_{g} g\right)=\sum t_{g} \varrho(g)$. The determinant of $\varrho\left(\sum t_{g} g\right)$ is a polynomial $F_{Q}(\mathbf{t})$ of degree $\operatorname{dim}(V)$ in the $m$ variables $\mathbf{t}=\left\{t_{g}\right\}_{g \in G}$ which is determined by and uniquely determines the isomorphism class of the representation $\varrho$. Thus the entire representation theory of $G$ can be expressed in terms of the "group determinants" $F_{Q}(\mathbf{t})$; this is in fact the way that representation theory was developed in its early years (see for instance Weber's Lehrbuch der Algebra, Vol. 2, Chap. 7).

If $V$ is reducible, say $V=V_{1} \oplus V_{2}$, then $F_{\varrho}(\mathbf{t})$ splits as $F_{Q_{1}}(\mathbf{t}) F_{Q_{2}}(\mathbf{t})$, so the study of group determinants can be reduced to the case of irreducible representations of $G$. At the other extreme, let $(V, R)$ be the (right) regular representation of $G$, i.e. $V=\mathbb{C}^{G}$ is the $m$-dimensional vector space of functions $f: G \rightarrow \mathbb{C}$ and $\varrho=R$ is given by

$$
(R(g) f)\left(g^{\prime}\right)=f\left(g^{\prime} g\right) \quad\left(g, g^{\prime} \in G\right) .
$$

The matrix representation of $R$ with respect to the basis of $\delta$-functions on $G$ is clearly given by

$$
R(g)_{g_{1}, g_{2}}= \begin{cases}1 & \text { if } g_{1} g=g_{2}, \\ 0 & \text { otherwise, }\end{cases}
$$

so that the group determinant $F_{R}(t)$ is the determinant of the $m \times m$ matrix $\left(t_{g_{1}^{-1}} g_{2}\right)_{g_{1}, g_{2} \in G}$. It is well known that $R$ contains every irreducible representation $\Pi$ of $G$ with positive multiplicity (equal to $\operatorname{dim} \Pi$ ). Hence if $F_{R}(\mathbf{t}) \neq 0$ for some $\mathbf{t} \in \mathbb{C}^{m}$ then $F_{I I}(\mathbf{t}) \neq 0$ for every irreducible representation $\pi$ and consequently $F_{\varrho}(\mathbf{t}) \neq 0$ for every representation $\varrho$ of $G$.

Now apply this to $G=\Theta_{n}, m=n$ !. Formula (4) and the discussion just given say that $A_{n}=A_{n}(q)$ is just the matrix representation $R\left(\alpha_{n}\right)$ of the element

$$
\alpha_{n}=\alpha_{n}(q)=\sum_{\pi \in \Im_{n}} q^{I(\pi)} \pi \in \mathbb{C}\left[\Im_{n}\right]
$$

acting on the regular representation $\left(\mathbb{C}^{\mathfrak{S}_{n}}, R\right)$. Here we are thinking of $q$ as being a complex number; if $q$ is thought of as a variable, then $\alpha_{n}(q)$ belongs to the group ring $\mathbb{Z}[q]\left[\Xi_{n}\right]$. We will usually consider $q$ as fixed and omit it from the notation. To prove Theorems 1 and 2, we will forget that $\alpha_{n}$ is acting on $\mathbb{C}^{\Xi_{n}}$ and simply show that it is invertible in the group algebra if $\prod_{i=1}^{n-1}\left(1-q^{k^{2}+k}\right) \neq 0$, in which case the inverse of the matrix $A_{n}$ is simply the matrix $R\left(\alpha_{n}^{-1}\right)$.

We now use this point of view to show how the positive definiteness of $A(q)=\left\{\left(\mathbf{x}_{\mathbf{1}}, \mathbf{x}_{\mathbf{k}}\right)\right\}$ follows from that of the $n ! \times n !$ matrices $A_{n}(q)$ for $n=1,2,3, \ldots$ Equation (1) gives by induction the formula for any indices $l, k_{1}, \ldots, k_{n}$ (not 
necessarily distinct)

$$
\begin{aligned}
a(l) a^{\dagger}\left(k_{1}\right) \cdots a^{\dagger}\left(k_{n}\right)= & q^{n} a^{\dagger}\left(k_{1}\right) \cdots a^{\dagger}\left(k_{n}\right) a(l) \\
& +\sum_{\substack{1 \leqq i \leqq n \\
k_{i}=l}} q^{i-1} a^{\dagger}\left(k_{1}\right) \cdots \widehat{a^{\dagger}\left(k_{i}\right)} \cdots a^{\dagger}\left(k_{n}\right),
\end{aligned}
$$

where the sum runs over those indices $i$ for which $k_{i}$ equals $l$ and the hat over the $i^{\text {th }}$ term of the product indicates that this term is to be omitted. Combining this with (2) gives

$$
a(l) a^{\dagger}\left(k_{1}\right) \cdots a^{\dagger}\left(k_{n}\right)|0\rangle=\sum_{\substack{1 \leqq i \leq n \\ k_{i}=\bar{l}}} q^{i-1} a^{\dagger}\left(k_{1}\right) \cdots \widehat{a^{\dagger}\left(k_{i}\right)} \cdots a^{\dagger}\left(k_{n}\right)|0\rangle .
$$

Now induction on $m$ gives a formula for $a\left(l_{m}\right) \cdots a\left(l_{1}\right) a^{\dagger}\left(k_{1}\right) \cdots a^{\dagger}\left(k_{n}\right)|0\rangle$ as a sum of terms $q^{N} a^{\dagger}\left(k_{1}\right) \cdots \widehat{a^{\dagger}\left(k_{i_{1}}\right)} \cdots \widehat{a^{\dagger}\left(k_{i_{m}}\right)} \cdots a^{\dagger}\left(k_{n}\right)|0\rangle$, where $i_{1}, \ldots, i_{m}$ are distinct indices with $k_{i_{1}}, \ldots, k_{i_{m}}$ equal to $l_{1}, \ldots, l_{m}$ in some order, the final result for $m=n$ being

$$
a\left(l_{n}\right) \cdots a\left(l_{1}\right) a^{\dagger}\left(k_{1}\right) \cdots a^{\dagger}\left(k_{n}\right)|0\rangle=\sum_{\substack{1 \leqq i_{1}, \ldots, i_{n} \leqq n \\ i_{1}, \ldots, i_{n} \text { distinct } \\ k_{i_{1}}=i_{1}, \ldots, k_{i_{n}}=l_{n}}} q^{\sharp\left\{1 \leqq r<s \leqq n, i_{r}>i_{s}\right\}}|0\rangle,
$$

i.e., in the notation of Sect. 1 ,

$$
\left(\mathbf{x}_{1}, \mathbf{x}_{\mathrm{k}}\right)=\left\langle 0\left|a\left(l_{n}\right) \cdots a\left(l_{1}\right) a^{\dagger}\left(k_{1}\right) \cdots a^{\dagger}\left(k_{n}\right)\right| 0\right\rangle=\sum_{\substack{\pi \in \Xi_{n} \\ l_{i}=k_{\pi(i)}(i=1, \ldots, n)}} q^{I(\pi)} .
$$

This formula includes (4) and also shows that $\left(\mathbf{x}_{\mathbf{l}}, \mathbf{x}_{\mathbf{k}}\right)=0$ unless $\mathbf{l}$ and $\mathbf{k}$ are permutations of one another, as already mentioned in Sect. 1, so that $A(q)$ splits up into the matrices $A_{\mathbf{k}_{0}}$ having as entries the numbers $\left(\mathbf{x}_{1}, \mathbf{x}_{\mathbf{k}}\right)$ for $\mathbf{l}$ and $\mathbf{k}$ ranging over all permutations of a given index set $\mathbf{k}_{0}$, e.g. for $\mathbf{k}_{\mathbf{0}}=(k, k, l)$ with $k \neq l$

$$
\begin{aligned}
A_{\mathbf{k}_{0}} & =\left(\begin{array}{lll}
\left(\mathbf{x}_{k k l}, \mathbf{x}_{k k l}\right) & \left(\mathbf{x}_{k k l}, \mathbf{x}_{k l k}\right) & \left(\mathbf{x}_{k k l}, \mathbf{x}_{l k k}\right) \\
\left(\mathbf{x}_{k l k}, \mathbf{x}_{k k l}\right) & \left(\mathbf{x}_{k l k}, \mathbf{x}_{k l k}\right) & \left(\mathbf{x}_{k l k}, \mathbf{x}_{l k k}\right) \\
\left(\mathbf{x}_{l k k}, \mathbf{x}_{k k l}\right) & \left(\mathbf{x}_{l k k}, \mathbf{x}_{k l k}\right) & \left(\mathbf{x}_{l k k}, \mathbf{x}_{l k k}\right)
\end{array}\right) \\
& =\left(\begin{array}{ccc}
1+q & q+q^{2} & q^{2}+q^{3} \\
q+q^{2} & 1+q^{3} & q+q^{2} \\
q^{2}+q^{3} & q+q^{2} & 1+q
\end{array}\right) .
\end{aligned}
$$

In each such matrix, the rows and columns are indexed by the permutations $\mathbf{k}=\pi \mathbf{k}_{0}$ of $\mathbf{k}_{\mathbf{0}}$ or equivalently by the left cosets $G / H$, where $G=\mathcal{\Xi}_{n}$ and $H$ is the subgroup of permutations of $\mathfrak{\Xi}_{n}$ fixing $\mathbf{k}_{\mathbf{0}}$. Write $\mathbf{k}=\sigma \mathbf{k}_{\mathbf{0}}, \mathbf{l}=\tau \mathbf{k}_{\mathbf{0}}$ with $\sigma, \tau \in \mathbb{S}_{n}$; then (10) says that the $(\mathbf{l}, \mathbf{k})$ matrix coefficient of $A_{\mathbf{k}_{0}}$ is equal to

$$
\sum_{\substack{\pi \in \Xi_{n} \\ \pi \sigma H=\tau H}} q^{I(\pi)}
$$

But a moment's thought shows that this is simply the $(\tau H, \sigma H)$-matrix coefficient (with respect to the basis of $\delta$-functions) of the element

$$
\alpha_{n}=\sum_{\pi} q^{I(\pi)} \pi
$$


on the subspace $V=\mathbb{C}^{G / H}$ of $\mathbb{C}^{G}$ consisting of functions $f: G \rightarrow \mathbb{C}$ which satisfy $f(g h)=f(g)$ for all $g \in G, h \in H$. This subspace is invariant under the action $R$ of $G$ on $\mathbb{C}^{G}$, so that $(V, R)$ is a representation of $G$. Hence if $\alpha_{n}$ is invertible in the group algebra $\mathbb{C}[G]$, then the matrix $A_{\mathbf{k}_{0}}$ is invertible. This completes the reduction of Theorem 1 to Theorem 2.

\section{Factorization of $\alpha_{n}$; Proof of Theorem 2}

We first introduce some notations. As in Sect. 1 we denote by $\left[i_{1}, i_{2}, \ldots, i_{n}\right]$ the permutation in $\Xi_{n}$ which sends 1 to $i_{1}, 2$ to $i_{2}, \ldots, n$ to $i_{n}$. We identify $\Xi_{n-1}$ with the subgroup of $\Xi_{n}$ consisting of permutations fixing $n$. For $1 \leqq k \leqq n$ we denote by $T_{k, n}$ the element $[1, \ldots, k-1, n, k, k+1, \ldots, n-1]$ of $\mathfrak{S}_{n}$, i.e.

$$
T_{k, n}(i)=\left\{\begin{array}{ll}
i & 1 \leqq i<k, \\
n & i=k, \\
i-1 & k<i \leqq n,
\end{array} \quad T_{k, n}^{-1}(i)= \begin{cases}i & 1 \leqq i<k, \\
i+1 & k \leqq i<n, \\
k & i=n .\end{cases}\right.
$$

Any element $\pi \in \Theta_{n}$ can be represented uniquely as $\sigma T_{k, n}$ with $\sigma \in \Theta_{n-1}$ and $1 \leqq k \leqq n$ (namely $k=\pi^{-1}(n), \sigma=\pi T_{k, n}^{-1}$ ), and a short calculation shows that then $I(\pi)$ equals $I(\sigma)+n-k$. Hence

$$
\alpha_{n}=\sum_{\pi \in \mathbb{S}_{n}} q^{I(\pi)} \pi=\sum_{\substack{\sigma \in \mathbb{E}_{n-1} \\ 1 \leqq k \leqq n}} q^{I\left(\sigma T_{k, n}\right)} \sigma T_{n, k}=\left(\sum_{\sigma \in \mathbb{E}_{n-1}} q^{I(\sigma)} \sigma\right)\left(\sum_{k=1}^{n} q^{n-k} T_{k, n}\right) .
$$

In other words,

Proposition 1. Define $\beta_{n}=\beta_{n}(q)=\sum_{k=1}^{n} q^{n-k} T_{k, n} \in \mathbb{C}\left[\mathfrak{S}_{n}\right]$. Then $\alpha_{n}=\alpha_{n-1} \beta_{n}$.

Here $\alpha_{n-1}$ is considered as an element of $\mathbb{C}\left[\mathfrak{S}_{n}\right]$ via the inclusion $\mathfrak{S}_{n-1} \subset \mathfrak{S}_{n}$. In particular, the representation of $\alpha_{n-1}$ in $R_{n}$, the $n$ !-dimensional regular representation of $\Xi_{n}$, consists of $n$ copies of the representation of $\alpha_{n-1}$ in $R_{n-1}$. Thus in terms of the matrices $A_{n}$ we can rewrite Proposition 1 as $A_{n}=\left(A_{n-1} \otimes 1_{n}\right) \cdot B_{n}$, where $A_{n-1} \otimes 1_{n}$ denotes the $n ! \times n$ ! block matrix with $n$ copies of $A_{n-1}$ on the diagonal blocks and zeros elsewhere and $B_{n}=B_{n}(q)$ has the matrix coefficients

$$
B_{n}(\pi, \sigma)= \begin{cases}q^{n-k} & \text { if } \pi \sigma^{-1}=T_{k, n} \text { for some } 1 \leqq k \leqq n, \\ 0 & \text { otherwise. }\end{cases}
$$

In particular, $\operatorname{det}\left(A_{n}(q)\right)=\operatorname{det}\left(A_{n-1}(q)\right)^{n} \operatorname{det}\left(B_{n}(q)\right)$, so by induction on $n$ we have reduced Theorem 2 to the simpler

Theorem 2'. $\operatorname{det}\left(B_{n}(q)\right)=\prod_{i=1}^{n-1}\left(1-q^{k^{2}+k}\right)^{\frac{n !}{k^{2}+k}}$.

We now make a second reduction by expressing $B_{n}$ in turn as a product of yet simpler matrices.

Proposition 2. For each $n$ define elements $\gamma_{n}, \delta_{n}$ in the group algebra $\mathbb{C}\left[\Theta_{n}\right]$ by

$$
\begin{aligned}
& \gamma_{n}=\left(1-q^{n-1} T_{1, n}\right)\left(1-q^{n-2} T_{2, n}\right) \cdots\left(1-q T_{n-1, n}\right), \\
& \delta_{n}=\left(1-q^{n+1} T_{1, n}\right)\left(1-q^{n} T_{2, n}\right) \cdots\left(1-q^{2} T_{n, n}\right) .
\end{aligned}
$$


Then $\beta_{n} \gamma_{n}=\delta_{n-1}$.

Proof. Let $\beta_{r, n}=\sum_{k=r}^{n} q^{n-k} T_{k, n}$, so that $\beta_{1, n}=\beta_{n}, \beta_{n, n}=1$ (note that $T_{n, n}=1 \in \Xi_{n}$ ).

Using the easily checked commutation relation

$$
T_{k, n} T_{r, n}=T_{r, n-1} T_{k+1, n} \quad(r \leqq k \leqq n-1),
$$

we find

$$
\begin{aligned}
\beta_{r, n} \cdot\left(1-q^{n-r} T_{r, n}\right)= & \sum_{k=r+1}^{n} q^{n-k} T_{k, n}+q^{n-r} T_{r, n} \\
& -q^{n-r} T_{r, n}-\sum_{k=r}^{n-1} q^{2 n-k-r} T_{k, n} T_{r, n} \\
= & \sum_{k=r+1}^{n} q^{n-k} T_{k, n}-\sum_{k=r+1}^{n} q^{2 n-k+1-r} T_{r, n-1} T_{k, n} \\
= & \left(1-q^{n-r+1} T_{r, n-1}\right) \cdot \beta_{r+1, n}
\end{aligned}
$$

and hence by induction on $r$ (starting with the trivial case $r=0$ )

$$
\begin{aligned}
& \beta_{1, n}\left(1-q^{n-1} T_{1, n}\right) \cdots\left(1-q^{n-r} T_{r, n}\right) \\
& \quad=\left(1-q^{n} T_{1, n-1}\right) \cdots\left(1-q^{n+1-r} T_{r, n-1}\right) \beta_{r+1, n} .
\end{aligned}
$$

The case $r=n-1$ of this identity is the desired identity.

To complete the proof of Theorem 2 we need to compute the determinants of the factors in $\gamma_{n}$ and $\delta_{n-1}$ under the regular representation $R_{n}$ of $\Xi_{n}$. We use the inclusions $\widetilde{S}_{b} \subset \Xi_{b+1} \subset \cdots \subset \mathfrak{S}_{n}$ to define elements $T_{a, b} \in \mathfrak{S}_{n}$ for all $1 \leqq a \leqq b \leqq n$ (we actually need only the cases $b=n-1$ and $n$ ). Its characteristic polynomial is given by:

Lemma. For $1 \leqq a \leqq b \leqq n$ the determinant of $R_{n}\left(1-t T_{a, b}\right)$ is $\left(1-t^{b-a+1}\right)^{\frac{n !}{b-a+1}}$.

Proof. The element $T_{a, b} \in \Theta_{n}$ is a cyclic permutation of the indices $a, a+1, \ldots, b$ and hence has order $b-a+1$. But if $G$ is an arbitrary finite group of order $m$ and $g \in G$ an element of order $d$, then the characteristic polynomial det $(1-t R(g))$ of $g$ under the regular representation is $\left(1-t^{d}\right)^{m / d}$, because the cycle structure of the permutation of $G$ given by left multiplication by $g^{-1}$ consists of $m / d$ disjoint cycles of length $d$. The lemma follows.

The proof of Theorem 2 is now immediate: we have

$$
\begin{aligned}
& \operatorname{det}\left(R_{n}\left(\gamma_{n}\right)\right)=\prod_{k=1}^{n-1} \operatorname{det}\left(R_{n}\left(1-q^{k} T_{n-k, n}\right)\right)=\prod_{k=1}^{n-1}\left(1-q^{k(k+1)}\right)^{\frac{n !}{k+1}}, \\
& \operatorname{det}\left(R_{n}\left(\delta_{n}\right)\right)=\prod_{k=1}^{n} \operatorname{det}\left(R_{n}\left(1-q^{k+1} T_{n-k+1, n}\right)\right)=\prod_{k=1}^{n}\left(1-q^{k(k+1)}\right)^{\frac{n !}{k}},
\end{aligned}
$$


and hence

$$
\begin{aligned}
\operatorname{det}\left(B_{n}\right)=\operatorname{det}\left(R_{n}\left(\beta_{n}\right)\right) & =\frac{\operatorname{det}\left(R_{n}\left(\delta_{n-1}\right)\right)}{\operatorname{det}\left(R_{n}\left(\gamma_{n}\right)\right)}=\frac{\operatorname{det}\left(R_{n-1}\left(\delta_{n-1}\right)\right)^{n}}{\operatorname{det}\left(R_{n}\left(\gamma_{n}\right)\right)} \\
& =\prod_{k=1}^{n-1}\left(1-q^{k(k+1)}\right)^{\frac{n \cdot(n-1) !}{k}-\frac{n !}{k+1}} \\
& =\prod_{k=1}^{n-1}\left(1-q^{k(k+1)}\right)^{\frac{n !}{k(k+1)}}
\end{aligned}
$$

which is Theorem 2'; Theorem 2 then follows by induction from this and Proposition 1.

\section{Formula for $A_{n}(q)^{-1}$}

According to Propositions 1 and 2 we have

$$
\begin{aligned}
& \beta_{n}=\delta_{n-1} \gamma_{n}^{-1} \\
& \alpha_{n}=\beta_{2} \cdots \beta_{n}=\delta_{1} \gamma_{2}^{-1} \delta_{2} \gamma_{3}^{-1} \cdots \gamma_{n-1}^{-1} \delta_{n-1} \gamma_{n}^{-1}
\end{aligned}
$$

and hence

$$
\alpha_{n}^{-1}=\gamma_{n} \delta_{n-1}^{-1} \gamma_{n-1} \cdots \gamma_{2} \delta_{1}^{-1}
$$

To invert $\alpha_{n}$, therefore, the first step is to invert $\delta_{k}$ for each $k$.

Proposition 3. For $\pi \in \Theta_{n}$ define $W(\pi) \in \mathbb{Z}$ by

$$
\begin{aligned}
W(\pi) & =\sum_{\substack{1 \leq i<j \leq n \\
\pi(i)>\pi(j)}}\left(1+(n+1-i)(n+1-j) \delta_{j-1, i}\right) \\
& =I(\pi)+\sum_{\substack{1 \leqq i \leqq n-1 \\
\pi(i+1)<\pi(i)}}(n+1-i)(n-\mathrm{i})
\end{aligned}
$$

and set $\varepsilon_{n}=\sum_{\pi \in \mathfrak{S}_{n}} q^{W(\pi)} \pi^{-1} \in \Im_{n}$. Then $\delta_{n}^{-1}=\frac{1}{\Delta_{n+1}} \varepsilon_{n}$ with $A_{n+1}$ as in Eq. (6).

Proof. Denote by $\sigma \mapsto \tilde{\sigma}$ the map $\Xi_{n-1} \rightarrow \Theta_{n}$ defined by $\tilde{\sigma}(1)=1$, $\tilde{\sigma}(i)=\sigma(i-1)+1$ for $i>1$ (this is a homomorphism since $\tilde{\sigma}$ is just $T_{1, n}^{-1} \sigma T_{1, n}$ ). Then $\tilde{T}_{a, b}=T_{a+1, b+1} \quad$ for $1 \leqq a<b \leqq n-1$, so $\delta_{n}=\left(1-q^{n+1} T_{1, n}\right) \widetilde{\delta}_{n-1}$. Hence by induction it suffices to show that $\varepsilon_{n}\left(1-q^{n+1} T_{1, n}\right)=\left(1-q^{n^{2}+n}\right) \tilde{\varepsilon}_{n-1}$.

For $\pi \in \mathbb{S}_{n}$, let $k=\pi^{-1}(1)$ and denote by $\pi^{\prime}$ the element $T_{1, n} \pi$ of $\Xi_{n}$. Since $\pi^{\prime}(k)=n$ but $\pi^{\prime}(i)=\pi(i)-1$ for all $i \neq k$, all the terms in the definition of $W(\pi)$ and of $W\left(\pi^{\prime}\right)$ are the same except those with $i$ or $j$ equal to $k$, so 


$$
\begin{aligned}
W\left(\pi^{\prime}\right)-W(\pi)= & \sum_{k<j \leqq n}\left(1+(n+1-k)(n+1-j) \delta_{j, k+1}\right) \\
& -\sum_{1 \leqq i<k}\left(1+(n+1-i)(n+1-k) \delta_{i, k-1}\right) \\
= & n-k+(n+1-k)(n-k)-(k-1) \\
& - \begin{cases}(n+1-k)(n+2-k) & \text { if } k>1 \\
0 & \text { if } k=1\end{cases} \\
= & \begin{cases}-n-1 & \text { if } k \neq 1, \\
n^{2}-1 & \text { if } k=1 .\end{cases}
\end{aligned}
$$

Hence

$$
\begin{aligned}
\varepsilon_{n}\left(1-q^{n+1} T_{1, n}\right) & =\sum_{\pi \in \Im_{n}}\left(q^{W(\pi)}-q^{W\left(T_{1, n} \pi\right)+n+1}\right) \pi^{-1} \\
& =\sum_{\substack{\pi \in \Xi_{n} \\
\pi(1)=1}}\left(q^{W(\pi)}-q^{W(\pi)+n^{2}+n}\right) \pi^{-1} \\
& =\left(1-q^{n^{2}+n}\right) \sum_{\sigma \in \mathbb{\Xi}_{n-1}} q^{W(\sigma)} \tilde{\sigma}^{-1}
\end{aligned}
$$

as desired.

We next give a formula expressing $\gamma_{n}$ as a sum rather than a product.

Proposition 4. The element $\gamma_{n} \in \Xi_{n}$ defined in Proposition 2 is given by

$$
\gamma_{n}=\sum_{k=1}^{n} \gamma_{n, k}, \quad \gamma_{n, k}=(-1)^{n-k} \sum_{\pi \in \mathfrak{\subseteq}_{n, k}} q^{I(\pi)} \pi^{-1},
$$

where $\Theta_{n, k}$ is the subset of $\Xi_{n}$ of cardinality $\left(\begin{array}{c}n-1 \\ k-1\end{array}\right)$ consisting of those permutations $\pi$ for which $\pi(1)<\cdots<\pi(k)>\cdots>\pi(n)$.

Proof. Multiplying out the terms in the product defining $\gamma_{n}$, we find

$$
\gamma_{n}=\sum_{s=0}^{n-1}(-1)^{s} \sum_{1 \leqq i_{1}<\ldots<i_{s} \leqq n-1} q^{\left(n-i_{1}\right)+\cdots+\left(n-i_{s}\right)} T_{i_{1}, n} T_{i_{2}, n} \cdots T_{i_{s}, n} .
$$

The element $\sigma=T_{i_{1}, n} T_{i_{2}, n} \cdots T_{i_{s}, n}$ of $\mathfrak{\subseteq}_{n}$ maps $i_{1}$ to $n, i_{2}$ to $n-1, \ldots$, and $i_{s}$ to $n-s+1$, and maps the rest of $\{1,2, \ldots, n\}$ monotone increasingly to $\{1,2, \ldots, n-s\}$. Moreover, it is easy to check that $\left(n-i_{1}\right)+\cdots+\left(n-i_{s}\right)$ equals $I(\sigma)$. The proposition now follows on setting $\pi=\sigma^{-1}$ and $k=n-s$.

The explicit formulas for $\delta_{n}^{-1}$ and $\gamma_{n}$ just given together with the formula $\alpha_{n}^{-1}=\gamma_{n} \delta_{n-1}^{-1} \alpha_{n-1}^{-1}$ give an inductive method to calculate $\alpha_{n}$ for each $n$. To describe this a little more explicitly, we define another element of $\mathbb{C}\left[\widetilde{\Xi}_{n}\right]$ by

$$
\zeta_{n}=\varepsilon_{n} \alpha_{n}^{-1}
$$

with $\varepsilon_{n}$ as in Proposition 3. We conjecture that $\zeta_{n}$ has coefficients which are polynomials in $q$. Propositions $1-3$ give $\alpha_{n}^{-1}=\Delta_{n}^{-1} \gamma_{n} \zeta_{n-1}$, so this conjecture implies the conjecture in (6). In fact, the two propositions are equivalent. Indeed, for each $\pi \in \Xi_{n, k}$ we have $\pi(k)=n$ and hence $\pi=\sigma T_{n, k}$ for some $\sigma \in \Xi_{n-1}$, so $\gamma_{n, k}$ 
equals $T_{n, k}^{-1} \gamma_{n-1, k}^{*}$ with $\gamma_{n-1, k}^{*} \in \mathbb{C}\left[\Xi_{n-1}\right]$ (in fact $\gamma_{n-1, k}^{*}=\gamma_{n-1, k-1}-\gamma_{n-1, k}$ ). It follows that if $\pi$ is any element of $\mathbb{S}_{n}$, and $\pi=\sigma T_{n, k}\left(1 \leqq k \leqq n, \sigma \in \mathbb{S}_{n-1}\right)$ its canonical decomposition as at the beginning of Sect. 3 , then the coefficient of $\pi$ in $\Delta_{n} \alpha_{n}^{-1}$ equals the coefficient of $\sigma^{-1}$ in $\gamma_{n-1, k}^{*} \zeta_{n-1}$. In particular, taking $k=n$ we find that the first $(n-1)$ ! coefficients in $\Delta_{n} \alpha_{n}^{-1}$ are exactly the coefficients of $\zeta_{n-1}$, so that the integrality of $\Delta_{n} \alpha_{n}$ implies that of $\zeta_{n-1}$ for each $n$.

We illustrate with numerical examples for $n \leqq 4$. For $n=2$ we have

$$
\begin{aligned}
& \alpha_{2}=1+q T_{1,2}, \quad \alpha_{2}^{-1}=\frac{1}{1-q^{2}}\left(1-q T_{1,2}\right), \quad \varepsilon_{2}=1+q^{3} T_{1,2}, \\
& \zeta_{2}=\varepsilon_{2} \alpha_{2}^{-1}=\left(1+q^{2}\right)-q T_{1,2} .
\end{aligned}
$$

We see that $\zeta_{2}$ is integral and that its coefficients are the first two coefficients of $\Delta_{3} \alpha_{3}^{-1}$, i.e., the first two coefficients of the matrix in (8). The other coefficients of $\alpha_{3}^{-1}$ are obtained by multiplying $\zeta_{2}$ by the elements $\gamma_{2, k}^{*}$ for $k=2$ and $k=3$, and we find

$$
\begin{aligned}
\left(1-q^{2}\right)\left(1-q^{6}\right) \alpha_{3}^{-1}= & \left(1-T_{2,3}^{-1}\left(q T_{1,3}+q^{2} T_{2,3}\right)+T_{1,3}^{-1}\left(q^{3} T_{1,3} T_{2,3}\right)\right) \zeta_{2} \\
= & \left(1+q^{2}\right)[123]-q[213]-q[132]-q^{4}[231] \\
& -q^{4}[312]+\left(q^{3}+q^{5}\right)[321],
\end{aligned}
$$

giving the remaining coefficients in the first row of the matrix in (8) (the other rows are permutations of the first one). Write this as $\Delta_{3} \alpha_{3}^{-1}=\left\{1+q^{2},-q,-q,-q^{4}\right.$, $\left.-q^{4}, q^{3}+q^{5}\right\}$ in the obvious notation. Using this value of $\alpha_{3}^{-1}$ and the value $\varepsilon_{3}=\left\{1, q^{7}, \quad q^{3}, q^{4}, \quad q^{8}, q^{11}\right\} \quad$ we find $\zeta_{3}=\left\{1+2 q^{2}+q^{4}+2 q^{6}+q^{8}\right.$, $\left.-q-q^{3}-q^{5}-q^{7},-q-q^{7},-q^{4},-q^{4}, q^{3}+q^{5}\right\}$, which is integral as claimed. Now multiplying this by the various components $\gamma_{4, k}(1 \leqq k \leqq 4)$, we find

$$
\begin{aligned}
& \alpha_{4}^{-1}=\left(1-q^{2}\right)^{-1}\left(1-q^{6}\right)^{-1}\left(1-q^{12}\right)^{-1} \\
& \quad \times\left\{1+2 q^{2}+q^{4}+2 q^{6}+q^{8},-q-q^{3}-q^{5}-q^{7},-q-q^{7},-q^{4},-q^{4}, q^{3}+q^{5}\right. \\
& \quad-q-q^{3}-q^{5}-q^{7}, q^{2}+q^{4}+q^{6},-q^{4},-q^{9}-q^{11}, 0, q^{10} ; \\
& \quad-q^{4}, 0, q^{3}+q^{5}, q^{10},-q^{8}-q^{10}-q^{12}, q^{7}+q^{9}+q^{11}+q^{13} \\
& \quad-q^{9}-q^{11}, q^{10}, q^{10}, q^{7}+q^{13}, q^{7}+q^{9}+q^{11}+q^{13} \\
& \left.\quad-q^{6}-2 q^{8}-q^{10}-2 q^{12}-q^{14}\right\}
\end{aligned}
$$

where the 24 components have been listed in the obvious order (namely, the elements $\sigma \in \mathcal{S}_{3}$ in the order above, followed by the elements $T_{3,4} \sigma$ with the same $\sigma$, then the $T_{2,4} \sigma$, then $\left.T_{1,4} \sigma\right)$. This gives the 24 elements of the first row of the matrix $A_{4}(q)^{-1}$, the other rows of course being permutations of this one. We have also checked the $\zeta_{4}$ has integral coefficients and thus that (6) holds for $n=5$.

\section{Number Operators}

For each index $k$, the $k^{\text {th }}$ number operator is the operator on $\mathbf{H}$ having each vector $\mathbf{x}_{\mathbf{k}}=a^{\dagger}\left(k_{1}\right) \cdots a^{\dagger}\left(k_{n}\right) \mid 0>$ as an eigenvector with eigenvalue equal to the number of $i$ with $k_{i}=k$, so that the eigenspace of $N(k)$ with eigenvalue $r$ is the space spanned 
by the states containing exactly $r$ particles of type $k$. It is easy to see that this definition is equivalent to the requirements

$$
N^{\dagger}(k)=N(k), \quad N(k)|0\rangle=0, \quad\left[N(k), a^{\dagger}(l)\right]=\delta_{k l} a^{\dagger}(l) \quad \text { for all } l
$$

(and hence $[N(k), a(l)]=-\delta_{k l} a(l)$ for all $l$ ).

Consider first the case in which there is only one operator $a(1)$ and its adjoint, i.e., only one kind of particle. In this case $\mathbf{H}(q)$ can be realized explicitly as the space spanned by vectors $e_{0}=|0\rangle, e_{1}, e_{2}, \ldots$ with

$$
a(1) e_{n}=\sqrt{n \frac{1-q^{n}}{1-q}} e_{n-1} \quad(=0 \text { for } n=0), \quad a^{\dagger}(1) e_{n}=\sqrt{\frac{1}{n+1} \frac{1-q^{n+1}}{1-q}} e_{n+1},
$$

since then

$$
a(1) a^{\dagger}(1) e_{n}-q a^{\dagger}(1) a(1) e_{n}=\frac{1-q^{n+1}}{1-q} e_{n}-q \frac{1-q^{n}}{1-q} e_{n}=e_{n},
$$

while the number operator $N(1)$ is given by either of the two formulas [5]

$$
N(1)=\sum_{n=1}^{\infty} \frac{(1-q)^{n}}{1-q^{n}} a^{\dagger}(1)^{n} a(1)^{n}=\sum_{n=1}^{\infty} \frac{(1-q)^{n}}{\log \left(1 / q^{n}\right)}\left(a^{\dagger}(1) a(1)\right)^{n},
$$

as one sees either by computing the action of the expressions on the right on the vectors $e_{n}$ or else by verifying the relations (11) using (1) and (2). The first formula makes sense for all $q$ between -1 and 1, the second (which can be rewritten $\left.\frac{1-q^{N(1)}}{1-q}=a^{\dagger}(1) a(1)\right)$ only for $0<q<1$. Both reduce to $N(1)=a^{\dagger}(1) a(1)$ in the limit as $q$ tends to 1 . For $q=0$ the first formula reduces to

$$
N(1)=\sum_{n=1}^{\infty} a^{\dagger}(1)^{n} a(1)^{n} \quad(q=0),
$$

which makes sense since only finitely many of the terms act non-trivially on any given state.

In [1], Greenberg showed that the generalization of (13) to the case when there are many indices $k$ is

$$
N(k)=\sum_{n=1}^{\infty} \sum_{k_{2}, \ldots, k_{n}} a^{\dagger}\left(k_{n}\right) \cdots a^{\dagger}\left(k_{2}\right) a^{\dagger}(k) a(k) a\left(k_{2}\right) \cdots a\left(k_{n}\right) \quad(q=0) .
$$

We now give a conjectural generalization of this formula to the case of arbitrary $q$ between -1 and 1. It is convenient to express the formula for all $N(k)$ simultaneously by giving a formula for the energy operator $\mathscr{E}=\sum_{k} E_{k} N(k)$, where the $E_{k}$ (interpreted as the energy of particle $k$ ) are scalar coefficients.

Conjecture. The operator $\mathscr{E}$ is given by

$$
\mathscr{E}=\sum_{n=1}^{\infty} \sum_{k_{1}, \ldots, k_{n}} \sum_{\pi \in \mathbb{E}_{n}} \sum_{i=1}^{n} c_{i}(q, \pi) E_{k_{i}} a^{\dagger}\left(k_{\pi(n)}\right) \cdots a^{\dagger}\left(k_{\pi(1)}\right) a\left(k_{1}\right) \cdots a\left(k_{n}\right),
$$


where the coefficients $c_{i}(q, \pi)$ are given $b y$

$$
\begin{aligned}
\sum_{\substack{\pi \in \Theta_{n} \\
1 \leqq i \leqq n}} c_{i}(q, \pi) X^{i-1} \pi= & \alpha_{n}^{-1}\left(1-q X T_{1,2}\right)\left(1-q^{2} X T_{1,3}\right) \\
& \cdots\left(1-q^{n-1} X T_{1, n}\right) \in \mathbb{C}[X]\left[\Xi_{n}\right] .
\end{aligned}
$$

This formula gives the correct result up to terms annihilating all 1-, 2-, and 3particle states, viz:

$$
\begin{aligned}
& \mathscr{E}=\sum_{k} E_{k} a^{\dagger}(k) a(k) \\
& +\frac{1}{1-q^{2}} \sum_{k, l}\left\{\left(E_{k}+q^{2} E_{l}\right) a^{\dagger}(l) a^{\dagger}(k)-q\left(E_{k}+E_{l}\right) a^{\dagger}(k) a^{\dagger}(l)\right\} a(k) a(l) \\
& +\frac{1}{\left(1-q^{2}\right)\left(1-q^{6}\right)} \times \\
& \sum_{k, l, m}\left\{\left(\left(1+q^{2}\right) E_{k}+\left(q^{2}+q^{6}\right) E_{l}+\left(q^{6}+q^{8}\right) E_{m}\right) a^{\dagger}(m) a^{\dagger}(l) a^{\dagger}(k)\right. \\
& \quad-q\left(E_{k}+E_{l}+q^{6} E_{m}\right) a^{\dagger}(m) a^{\dagger}(k) a^{\dagger}(l) \\
& \quad-q\left(E_{k}+q^{6}\left(E_{l}+E_{m}\right)\right) a^{\dagger}(l) a^{\dagger}(m) a^{\dagger}(k) \\
& \quad-q^{4}\left(E_{k}+E_{l}+E_{m}\right) a^{\dagger}(k) a^{\dagger}(m) a^{\dagger}(l) \\
& \quad-q^{4}\left(E_{k}+E_{l}+E_{m}\right) a^{\dagger}(l) a^{\dagger}(k) a^{\dagger}(m) \\
& \left.\quad+q^{3}\left(1+q^{2}\right)\left(E_{k}+E_{l}+E_{m}\right) a^{\dagger}(k) a^{\dagger}(l) a^{\dagger}(m)\right\} a(k) a(l) a(m) \\
& +\ldots
\end{aligned}
$$

Note added in proof. The conjecture stated in this section has now been proved by Sonia Stanciu (see paper following this one).

\section{References}

1. Greenberg, O.W.: Example of infinite statistics. Phys. Rev. Lett. 64, 705-708 (1990)

2. Biedenharn, L.C.: J. Phys. A22, L873-L878 (1989)

3. Fivel, D.: Interpolation between Fermi and Bose statistics using generalized commutators. Phys. Rev. Lett. 65, 3361-3364 (1990)

Bożejko, M., Speicher, R.: Commun. Math. Phys. 137, 519-531 (1991)

4. Greenberg, O.W.: Q-mutators and violations of statistics. Argonne Workshop on Quantum Groups. T. Curtright, D. Fairlie, C. Zachos (eds.). Singapore: World Scientific, pp. 166-180 (1991)

Greenberg, O.W.: Particles with small violations of Fermi or Bose statistics. Phys. Rev. D 43, $4111-4120(1991)$

Greenberg, O.W.: Interactions of particles having small violations of statistics. Physica A (to appear)

5. Freund, P.G.O., Nambu, Y.: Private communication (1989) 